\title{
Impact of choice of imaging modality accompanying outpatient exercise stress testing on outcomes and resource use after revascularization for acute coronary syndromes
}

\author{
Jerome J. Federspiel, PhD ${ }^{a, b, c}$, Bimal R. Shah, MD, MBA ${ }^{a, d}$, Leslee J. Shaw, PhD ${ }^{e}$, \\ Frederick A. Masoudi, MD, MSPH ${ }^{f}$, Patricia P. Chang, MD, MHS ${ }^{c}$, Sally C. Stearns, $\mathbf{P h D}^{\mathrm{b}}$, \\ Daniel W. Mudrick, MD, MPH ${ }^{\mathrm{d}, \mathrm{g}}$, Patricia A. Cowper, PhDa , Cynthia L. Green, PhD ${ }^{\mathrm{a}}$, and \\ Pamela S. Douglas, MD ${ }^{a, d}$ \\ aDuke Clinical Research Institute, Durham, NC \\ bUNC Gillings School of Global Public Health, Chapel Hill, NC \\ cUniversity of North Carolina School of Medicine, Chapel Hill, NC \\ dDuke University School of Medicine, Durham, NC \\ eEmory University, Atlanta, GA \\ ${ }^{\mathrm{f} A n s c h u t z}$ Medical Campus, University of Colorado, Aurora, $\mathrm{CO}$ \\ gMcConnell Heart Health Center, Columbus, $\mathrm{OH}$
}

\begin{abstract}
Background-Exercise stress testing is commonly obtained after percutaneous coronary intervention (PCI) performed for acute coronary syndromes (ACS). We compared the relationships between exercise echocardiography and nuclear testing after ACS-related PCI on outcomes and resource use.
\end{abstract}

\begin{abstract}
Methods-Longitudinal observational study using fee-for-service Medicare claims to identify patients undergoing outpatient exercise stress testing with imaging within 15 months after PCI performed for ACS between 2003 and 2004.
\end{abstract}

\begin{abstract}
Results-Of 63,100 patients undergoing stress testing 3 to 15 months post-PCI, 31,731 (50.3\%) underwent an exercise stress test with imaging. Among 29,279 patients undergoing exercise stress testing with imaging, $15.5 \%$ received echocardiography. Echocardiography recipients had higher rates of repeat stress testing (adjusted hazard ratio [HR] 2.60, CI 2.19-3.10) compared with those undergoing nuclear imaging in the 90 days after testing, but lower rates of revascularization (adjusted HR 0.87, CI 0.76-0.98) and coronary angiography (adjusted HR 0.88, CI 0.80-0.97).
\end{abstract}

() 2013, Mosby, Inc. All rights reserved.

Reprint requests: Pamela S. Douglas, MD, Duke Clinical Research Institute, 2400 Pratt St, Durham, NC 27703. pamela.douglas@duke.edu.

Disclosures

This study was sponsored by the Agency for Healthcare Research and Quality, US Department of Health and Human Services, Rockville, MD, as part of the Cardiovascular Consortium and funded under project 24-DKE-3 and work assignment number HHSA290-2005-0032-I-TO4-WA3 as part of the Developing Evidence to Inform Decisions about Effectiveness program. The authors of this manuscript are responsible for its content. Statements in the manuscript should not be construed as endorsement by US Department of Health and Human Services.

This study was also supported by the National Heart, Lung, and Blood Institute (F30-HL110483), the National Institute for General Medical Sciences (T32-GM008719), and the Agency for Healthcare Research and Quality (K12-HS19479). This manuscript's contents are the responsibility of the authors and do not necessarily represent the views of the National Institutes of Health or the Agency for Healthcare Research and Quality. 
None of these differences persisted subsequent to 90 days after stress testing. Rates of death and readmission for myocardial infarction rates were similar. Total Medicare payments were lower initially after echocardiography (incremental difference \$498, CI 488-507), an effect attributed primarily to lower reimbursement for the stress test itself, but not significantly different after 14 months after testing.

Conclusions-In this study using administrative data, echocardiography recipients initially had fewer invasive procedures but higher rates of repeat testing than nuclear testing recipients. However, these differences between echo and nuclear testing did not persist over longer time frames.

Exercise stress testing is commonly performed after percutaneous coronary intervention (PCI), ${ }^{1}$ frequently accompanied with nuclear or echocardiography imaging. ${ }^{2}$ Optimal testing approaches for patients without diagnosed coronary artery disease presenting with chest pain have been previously examined; however, little is known about optimal stress testing strategies in patients who have undergone revascularization. ${ }^{3,4}$ Understanding the value of alternative testing modalities is critical, given the costs of cardiac imaging-related services in the United States. ${ }^{5}$

To date, most evaluations of imaging have focused narrowly on test performance parameters such as sensitivity and specificity; only recently has the research agenda been expanded to the comparative value of imaging strategies. ${ }^{6}$ Unfortunately, studies of imaging value have typically focused only on the testing costs, rather than the impact of testing on overall resource use during or after episodes of care. Health care payment reforms have begun providing incentives to facilitate transition from fee-for-service reimbursement to other payment approaches, such as bundling or accountable care organizations. These changes increase the importance of understanding the implications of test choices on resource use. ${ }^{7}$ We used longitudinal administrative data to compare outcomes and resource use after PCI among patients with acute coronary syndromes (ACS), an indication for which PCI is commonly performed.

\section{Methods}

\section{Data sources and subjects}

Medicare Provider Analysis and Review files from 2003 and 2004 were obtained for discharges with International Classification of Diseases, Ninth Edition, Clinical Modification (ICD-9-CM) diagnosis codes for myocardial infarction (410.xx), unstable angina (411.1), or angina not otherwise specified (403.9). The first admission for each patient was considered their index stay. The sample was restricted to patients (1) treated at a short stay facility and (2) who did not have a diagnosis code reflecting a history of PCI, coronary artery bypass grafting $(\mathrm{CABG})$, or valve replacement, to generate a population of patients treated acutely for ACS and who had not yet undergone coronary revascularization or cardiac surgery to create a cohort treated immediately for ACS and who had not yet undergone previous revascularization or cardiac surgery. Next, Denominator files were obtained and used to apply additional exclusions, including (1) living outside the 50 US States and Washington, DC; (2) not continuously enrolled in fee-for-service Medicare rather than managed care plans (both exclusions to ensure complete measurement of subsequent outcomes and resource use); and (3) younger than age 66 years (to allow for a full year of claims data before the index stay). Owing to limitations on cohort size imposed by the Centers for Medicare and Medicaid Services (CMS), patients discharged after October 14, 2004, were excluded to reduce the cohort to $1,000,000$ beneficiaries. 
For this study, the cohort was further restricted to individuals admitted for ACS. For all such patients, 2003 to 2008 inpatient, outpatient, and carrier claims were obtained to determine whether patients received coronary revascularization (CABG or PCI) in the 30 days subsequent to their index stay and, if performed, to characterize the revascularization procedures performed. To ensure complete characterization of revascularization, we excluded patients whose revascularization claims did not list Medicare as the primary payer or for whom it was not possible to link either an inpatient or outpatient claim for the facility charge component of the revascularization to a Carrier claim for the professional component. Figure 1A summarizes initial data set construction, whereas online Appendix Supplementary Table I lists the coding criteria.

\section{Stress test population}

Ultimately, 162,904 PCI recipients were included for analysis (Figure 1B). We defined a 60day "blackout period" after each patient's index event because diagnostic tests during this period may be performed for cardiac rehabilitation, staging revascularization, or functional assessment. ${ }^{1}$ Any stress testing during this period was ignored. We excluded patients who died $(n=19,789)$, readmitted for myocardial infarction $(n=2,169)$, or underwent repeat revascularization or coronary angiography $(n=15,161)$ during the blackout period.

Among the remaining 135,785 patients, we identified patients who received an outpatient stress test between 2 and 15 months after their index PCI event that was not preceded by coronary angiography, repeat revascularization, or myocardial infarction readmission. Each patient's first eligible stress test was included in the analysis. We excluded patients (1) receiving an inpatient stress test (based on place of service codes) because of an inability to identify pharmacologic stress agents on inpatient claims, (2) receiving positron emission tomography or magnetic resonance imaging because these tests were rarely performed, (3) who were coded as having both stress nuclear and echocardiography procedures on the same day, (4) receiving an electrocardiogram-only test, and (5) who received pharmacologic stress testing, as pharmacologic testing was overwhelmingly ( $\sim 98 \%)$ performed with nuclear imaging. Ultimately, 29,279 eligible patients were included in the comparison of echocardiographic and nuclear stress testing.

\section{Treatment and outcome definitions}

The use of stress testing after PCI was identified by Healthcare Common Procedure Coding System (HCPCS) codes. Electrocardiogram stress and nuclear imaging procedures performed within 1 day of each other were considered a single nuclear test; similarly, electrocardiogram stress and echocardiographic testing performed on the same day were considered a single echocardiography test.

We considered 6 outcomes: all-cause mortality, readmission for myocardial infarction, coronary revascularization, coronary angiography, additional stress testing, and Medicare payments. The number and dates of coronary angiography and coronary revascularization (either PCI or CABG) procedures after stress testing were identified using ICD-9-CM procedure, HCPCS, and diagnosis-related grouping (DRG) codes. Medicare payments were obtained by measuring total payments on inpatient, outpatient, and carrier claims occurring on and/or after the stress testing date. In addition to overall payments, we also evaluated payments stratified by whether the claim included a stress test-related line item.

\section{Other covariates}

The presence of comorbid conditions was measured using Elixhauser criteria, based on diagnosis codes recorded on claims in the year preceding the index ACS event. ${ }^{8}$ Because diagnosis codes on outpatient and carrier claims are not always validated, comorbidities 
recorded on these claims were only included if they were documented on 2 or more claims for dates of service 30 or more days apart. ${ }^{9}$ Percutaneous coronary intervention facility characteristics were obtained from Medicare Provider of Services Files. Small area socioeconomic data, measured at the ZIP code level, were obtained from the 2000 US Census. ${ }^{10}$

\section{Statistical analysis}

We present percentages and medians with interquartile ranges (IQRs) for categorical and continuous variables, respectively. Patient characteristics were compared between imaging modalities using $\chi^{2}$ tests for categorical and Kruskal-Wallis tests for continuous variables.

A propensity score model, estimating the probability of receiving echocardiography versus nuclear imaging, was constructed using a logistic regression. Covariates (online Appendix Supplementary Table I and online Appendix Supplementary Figure 2) were selected based on factors that may confound the relationship between imaging modality and outcomes ${ }^{11}$ including the following: demographics, socioeconomic characteristics, PCI and PCI facility characteristics, time from PCI to stress testing, Medicare payments in the 60 days preceding stress testing, and whether the patient received care for comorbid conditions in the year before their index stay. Inverse probability weighting was used to adjust for differences in baseline characteristics. ${ }^{12} \mathrm{We}$ evaluated covariate balance before and after inverse probability weighting using standardized differences. ${ }^{13}$

All time-to-event outcomes were analyzed treating death as a competing risk, and end of fee-for-service claims availability was a censoring event. For the coronary angiography and revascularization outcomes, readmission for myocardial infarction was considered an additional competing risk; for repeat stress testing, readmission for myocardial infarction, revascularization, and coronary angiography were all considered competing events. Unadjusted results were portrayed using cumulative incidence curves, and bivariate tests of association were performed between imaging modalities using Gray test. ${ }^{14}$ Adjusted hazard ratios (HRs) were generated using cause-specific Cox proportional hazards models, adjusted using inverse probability weighting. To determine whether imaging modality affected shortterm processes of care differently than long-term processes, we constructed models with interaction terms between imaging modality and time (a binary indicator for time being within 90 days after testing). The 90-day threshold was specified a priori to distinguish "short-term" and "long-term" processes of care; however, we conducted sensitivity analyses with thresholds set at 30-day increments between 30 and 180 days. We also evaluated an alternative model formulation modeling cumulative incidence in the presence of competing risks, rather than cause-specific hazards. ${ }^{15}$

CMS payments were estimated in a partitioned framework to allow for censoring caused by differential follow-up length. ${ }^{16}$ A person-period data set was constructed using 1 period for the day of stress testing followed by up to 40 additional 30-day periods. Each patient's resource use was included for all periods in which they were completely observed. Adjusted estimates of the difference in cost were constructed using inverse propensity weighted (IPW)-adjusted linear models; clustered SDs were used to account for within-person correlation in the person-period observations. ${ }^{17}$

Statistical analyses were conducted using SAS version 9.2 (SAS Institute, Inc, Cary, NC), R version 2.11.1 (R Foundation, Vienna, Austria), and Stata/MP version 12.1 (Statacorp, College Station, TX) with a 2 -sided $a$ level of .05 prespecified as significant. The University of North Carolina at Chapel Hill institutional review board granted a waiver of the informed consent and authorization for this study. This study was supported by the Agency for Healthcare Research and Quality and the National Institutes of Health. The authors are 
solely responsible for the design and conduct of this study, study analyses, the drafting and editing of the manuscript, and its final contents.

\section{Results}

\section{Characteristics of study population}

Of 29,279 eligible patients (Figure 1), 4,542 (15.5\%) received exercise echocardiography. Patients receiving exercise echocardiography had fewer comorbid conditions (eg, heart failure, pulmonary circulatory disease, peripheral vascular disease, chronic lung disease, and diabetes mellitus) than those receiving exercise nuclear testing (Table I). Patients receiving exercise echocardiography were also more likely to have received a drug-eluting (rather than bare-metal) coronary stent during their index event. There were no differences in the rates of the various ACS diagnoses. Echocardiography patients had received PCI during their index event in smaller facilities.

\section{Propensity score model}

Among echocardiography recipients, propensity scores ranged from 0.02 to 0.51 , with median 0.16 (IQR 0.12-0.25); among nuclear imaging recipients, propensity scores ranged from ranged from 0.02 to 0.51 , with a median of 0.13 (IQR 0.10-0.17) (online Appendix Supplementary Figure 1). Virtually all (99.9\%) observations fell in the region of common support of the propensity score, suggesting that the analysis does not entail comparison of distinct populations. The initially selected logistic regression model, constructed without interactions or polynomial terms, produced excellent covariates balance, evidenced by reductions in standardized differences after inverse probability weighting (online Appendix Supplementary Figure 2).

\section{Outcomes and resource use}

Follow-up data were available for a median of 1,666 days after stress testing (IQR 1,4461,854 days) and were identical for echocardiography and nuclear testing (1,667 vs 1,666 days, $P=.81$ ). In unadjusted analyses, patients receiving exercise echocardiography had a lower incidence of diagnostic angiography and repeat revascularization after testing than did exercise nuclear patients but higher rates of repeat stress testing (Figure 2). The incidence of coronary angiography was higher for nuclear than echocardiography recipients at 90 days (14.9\% vs $12.7 \%$ ) and 3 years (34.8\% vs $32.0 \%)$; nuclear patients also had a higher incidence of repeat revascularization at both 90 days (9.0\% vs $7.7 \%)$ and 3 years $(20.0 \%$ vs $18.7 \%$ ). Echocardiography patients had more repeat stress tests at 90 days after testing $(5.0 \%$ vs $1.8 \%)$ and at 3 years $(60.3 \%$ vs $55.7 \%)$. Of particular note was a sharp uptick in repeat stress test usage approximately 1 year after stress testing, suggesting common use of scheduled annual stress testing.

In adjusted analyses (Table II), overall rates of coronary angiography were lower in exercise echocardiography recipients (adjusted HR 0.93, 95\% CI 0.88-0.98), but rates of repeat stress testing (adjusted HR 1.07, 95\% CI 1.03-1.12) were higher. Differences in rates of death, admission for subsequent myocardial infarction, and repeat revascularization were not statistically significant. After the first 90 days after testing, rates of all events were equal in patients tested with echocardiography and nuclear imaging. Rates of death and admission for myocardial infarction were not significantly different overall. Owing to the very low $(<1 \%)$ incidence of both outcomes in the 90 days following stress testing, results are not reported separately by time cut point for these outcomes. Results were consistent across a range of cut points of 30 to 180 days after stress testing (online Appendix Supplementary Table I) and when estimated using competing risk models (online Appendix Supplementary Table II). 
Without adjustment, exercise echocardiography was associated with lower total Medicare payments than exercise nuclear on the day of stress testing (incremental difference \$-497.61, CI -506.89 to -488.32); the cumulative difference between exercise echocardiography and exercise nuclear grew throughout the study period (Figure 3). After IPW adjustment, the difference in payments on day of test was comparable with the unadjusted estimate (incremental difference $-\$ 499.42$, CI -510.34 to -488.51 ); however, the cumulative payment difference between echocardiography and nuclear imaging decreased over time and was no longer statistically significant at 14 months after stress testing. Stratifying payments into those for stress testing versus all other services illustrated that stress testing-related payments were lower for exercise echocardiography recipients, but there was no significant difference in payments for all other services (Figure 4). The $\$ 499.42$ difference in overall payments observed on the day of initial stress test was explained by differences in spending for stress testing-related services (\$509.04). The difference in cumulative stress testingrelated payments grew smaller during the first year after the initial testing but increased in magnitude for time points subsequent to 1 year.

\section{Discussion}

In a national cohort of patients older than 65 years with ACS undergoing exercise stress testing with imaging after coronary stenting, patients tested with echocardiography and nuclear imaging had similar rates of death and myocardial infarction after adjustment for baseline differences; however, the pattern of resource use after stress testing differed. Echocardiography recipients had higher short-term rates of repeat stress testing but lower rates of invasive testing and intervention. Moreover, costs differed depending on the time interval considered, with total CMS payments being lower among echocardiography recipients immediately after testing, but not significantly different after 14 months after testing, with this difference explained by the difference in charges from the initial stress test.

Although the baseline clinical characteristics of patients tested with echocardiography versus nuclear imaging demonstrated only modest differences, patients tested with echocardiography had a lower burden of risk factors than those tested with nuclear testing, including lower rates of most comorbidities. Such differences have been suggested in previous research but have generally not been carefully examined despite their potential to skew conclusions based on Bayesian principles. ${ }^{18,19}$ We attempted to adjust for confounding using propensity score techniques.

The results observed in our study may reflect differences in test characteristics between the 2 modalities. When compared with nuclear imaging, echocardiography has been demonstrated to be a less sensitive but more specific test because wall motion abnormalities are "further down the ischemic cascade" than ischemia detectable by nuclear imaging. ${ }^{20,21}$ These performance characteristics may contribute to lower short-term use of coronary angiography and revascularization in post-PCI patients receiving echocardiographic testing, a finding that has been previously demonstrated for patients without a history of coronary artery disease. ${ }^{3}$ The dissipation of this effect after stress testing may indicate how quickly patients move through an episode of care.

Stress echocardiography resulted in higher short-term rates of repeat stress testing than did nuclear imaging. Several factors may explain this finding. Because stress echocardiography studies are sometimes viewed as more challenging to interpret than nuclear images, higher short-term rates of repeat testing may reflect a higher rate of equivocal studies. Because physician confidence in test results is inversely correlated with rates of additional test use, our findings may also reflect lower physician comfort with echocardiography findings. ${ }^{22}$ Finally, nuclear stress testing may be viewed as having a longer "warranty period" (ie, the 
period after a normal study in which the patient is viewed as being highly unlikely to have recurrent ischemia) than echocardiography. ${ }^{23}$ Because the effect attributed to echocardiography was stronger in the short term than the long term, an initial lack of confidence or clarity in test results (which would drive greater use of immediate repeat testing) seems a more relevant factor than differences in warranty period.

Most outpatient stress testing after PCI is used either to assess symptoms suggestive of recurrent/progressive myocardial ischemia or to screen asymptomatic patients. Evidence suggests that revascularization procedures in patients with stable chest pain may improve quality of life, but generally do not improve survival or prevent myocardial infarction. ${ }^{24}$ Thus, our finding of similar rates of death and myocardial infarction (regardless of stress test imaging modality used) is consistent with the overall good prognosis of this patient population. Furthermore, documentation of similar clinical outcomes makes differences in resource use a more important consideration. We found that stress echocardiography was associated with lower CMS payments on the day of, and up to 14 months after, initial testing. After 14 months, the difference in CMS payments was no longer significant. The difference in test reimbursement for echocardiography versus nuclear imaging during the period under study (2003-2005) appeared to be the primary determinant of cost difference rather than downstream service use, suggesting that equalization of reimbursement for echocardiography and nuclear testing would result in similar long-term costs to payers. The pattern also suggests that careful attention to decisions regarding invasive workup shortly after stress testing may be a promising approach to controlling cost without affecting outcome, in light of the similarities in death and myocardial infarction rates.

In aggregate, our findings illustrate complexities when evaluating cardiac imaging and using results to inform clinical practice and health policy. The differences observed in the populations chosen for each test suggest that careful attention to the risk profile of cohorts under study is needed to accurately compare testing strategies. This assessment may be accomplished through careful statistical adjustment or by randomization, as has been done in a handful of studies, including the recently completed What Is the Optimal Method for Ischemia Evaluation in Women (WOMEN) trial $^{4}$ and the ongoing PROspective Multicenter Imaging Study for Evaluation of Chest Pain (PROMISE) study. The differences in downstream testing and procedure rates but similar rates of outcomes-even after statistical adjustment for clinical and other characteristics-demonstrate the need to examine parameters other than diagnostic and prognostic accuracy when evaluating testing strategies. In general, outcome studies for cardiac imaging should consider the short- and long-term impact on processes of care and resource use. ${ }^{6}$ This effort may be complicated by the potential for "tradeoffs" associated with test choice, as observed here. Furthermore, length of follow-up is also an important consideration. If only test reimbursement is considered, exercise echocardiography might appear less expensive, whereas if the longer term is considered, there may be little difference between imaging modalities. As payment models transition toward payment for episodes of care rather than on a fee-for-service basis, episode length (as well as testing during the 60-day blackout period excluded from this study) may play an important role in what are perceived to be optimal testing strategies.

Our study had several limitations. First, the study cohort was limited to patients older than 65 years and enrolled in fee-for-service Medicare. The results may not apply to other populations. This study focuses on the use of stress testing in outpatient settings, and results may not apply to inpatient stress testing. There is potential for residual confounding between imaging modality and outcome because of unmeasured patient characteristics. Although we were able to measure whether patients received drug-eluting stents and whether they underwent a multivessel procedure, detailed angiographic characteristics were not available. Finally, we lacked detailed clinical data (eg, symptoms) at the time of stress testing. As a 
result, we cannot identify the indication for which the stress test was ordered and, therefore, cannot examine stress testing appropriateness.

In summary, the choice of using echocardiography versus nuclear imaging in conjunction with exercise stress testing in patients who have received PCI for ACS results in a different pattern of subsequent care. Echocardiography recipients receive fewer invasive procedures in the short term but face increased use of repeat stress testing. Conversely, nuclear stress testing results in higher rates of downstream coronary angiography and repeat revascularization. These differences in posttesting patterns of care highlight that analyses of imaging value must consider not only unit cost and performance characteristics but also how initial testing choice affects outcomes and processes of care. Such considerations will be relevant for policy makers and providers in designing new reimbursement schemes for patients with ACS.

\section{Supplementary Material}

Refer to Web version on PubMed Central for supplementary material.

\section{References}

1. Shah BR, Cowper PA, O'Brien SM, et al. Patterns of cardiac stress testing after revascularization in community practice. J Am Coll Cardiol. 2010; 56(16):1328-34. [PubMed: 20888523]

2. Federspiel JJ, Mudrick DW, Shah BR, et al. Patterns and predictors of stress testing modality after percutaneous coronary stenting: data from the $\operatorname{NCDR}((\mathrm{R}))$. JACC Cardiovasc Imaging. 2012; 5(10): 969-80. [PubMed: 23058063]

3. Shreibati JB, Baker LC, Hlatky MA. Association of coronary CT angiography or stress testing with subsequent utilization and spending among Medicare beneficiaries. JAMA. 2011; 306(19):2128-36. [PubMed: 22089720]

4. Shaw LJ, Mieres JH, Hendel RH, et al. Comparative effectiveness of exercise electrocardiography with or without myocardial perfusion single photon emission computed tomography in women with suspected coronary artery disease: results from the What Is the Optimal Method for Ischemia Evaluation in Women (WOMEN) trial. Circulation. 2011; 124(11):1239-49. [PubMed: 21844080]

5. Shaw LJ, Marwick TH, Zoghbi WA, et al. Why all the focus on cardiac imaging? JACC Cardiovasc Imaging. 2010; 3(7):789-94. [PubMed: 20633864]

6. Douglas PS, Taylor A, Bild D, et al. Outcomes research in cardiovascular imaging: report of a workshop sponsored by the National Heart, Lung, and Blood Institute. JACC Cardiovasc Imaging. 2009; 2(7):897-907. [PubMed: 19608141]

7. Cutler DM, Ghosh K. The potential for cost savings through bundled episode payments. N Engl J Med. 2012; 366(12):1075-7. [PubMed: 22435368]

8. Elixhauser A, Steiner C, Harris DR, et al. Comorbidity measures for use with administrative data. Med Care. 1998; 36(1):8-27. [PubMed: 9431328]

9. Glaser R, Selzer F, Faxon DP, et al. Clinical progression of incidental, asymptomatic lesions discovered during culprit vessel coronary intervention. Circulation. 2005; 111(2):143-9. [PubMed: 15623544]

10. US Census Bureau. Census 2000 Summary File 3-United States. Washington, DC: US Department of Commerce; 2002.

11. Brookhart MA, Schneeweiss S, Rothman KJ, et al. Variable selection for propensity score models. Am J Epidemiol. 2006; 163(12):1149-56. [PubMed: 16624967]

12. Hirano K, Imbens GW. Estimation of causal effects using propensity score weighting: an application to data on right heart catheterization. Health Serv Outcomes Res Methodol. 2001; 2:259-78.

13. Austin PC. Primer on statistical interpretation or methods report card on propensity-score matching in the cardiology literature from 2004 to 2006: a systematic review. Circ Cardiovasc Qual Outcomes. 2008; 1(1):62-7. [PubMed: 20031790] 
14. Gray RJ. A class of K-sample tests for comparing the cumulative incidence of a competing risk. Ann Stat. 1988; 16(3):1141-54.

15. Fine JP, Gray RJ. A proportional hazards model for the subdistribution of a competing risk. J Am Stat Assoc. 1999; 94(446):496-509.

16. Bang H, Tsiatis AA. Estimating medical costs with censored data. Biometrika. 2000; 87(2):329_ 43.

17. White H. A heteroskedasticity-consistent covariance matrix estimator and a direct test for heteroskedasticity. Econometrica. 1980; 48(4):817-38.

18. Shaw LJ, Marwick TH, Berman DS, et al. Incremental cost-effectiveness of exercise echocardiography vs. SPECT imaging for the evaluation of stable chest pain. Eur Heart J. 2006; 27(20):2448-58. [PubMed: 17003046]

19. Bart BA, Erlien DA, Herzog CA, et al. Marked differences between patients referred for stress echocardiography and myocardial perfusion imaging studies. Am Heart J. 2005; 149(5):888-93. [PubMed: 15894973]

20. Heijenbrok-Kal MH, Fleischmann KE, Hunink MG. Stress echocardiography, stress single-photonemission computed tomography and electron beam computed tomography for the assessment of coronary artery disease: a meta-analysis of diagnostic performance. Am Heart J. 2007; 154(3): 415-23. [PubMed: 17719283]

21. Schinkel AF, Bax JJ, Geleijnse ML, et al. Noninvasive evaluation of ischaemic heart disease: myocardial perfusion imaging or stress echocardiography? Eur Heart J. 2003; 24(9):789-800. [PubMed: 12727146]

22. Adriaensen ME, Kock MC, Stijnen T, et al. Peripheral arterial disease: therapeutic confidence of CT versus digital subtraction angiography and effects on additional imaging recommendations. Radiology. 2004; 233(2):385-91. [PubMed: 15358853]

23. Hachamovitch R, Hayes S, Friedman JD, et al. Determinants of risk and its temporal variation in patients with normal stress myocardial perfusion scans: what is the warranty period of a normal scan? J Am Coll Cardiol. 2003; 41(8):1329-40. [PubMed: 12706929]

24. Boden WE, O'Rourke RA, Teo KK, et al. Optimal medical therapy with or without PCI for stable coronary disease. N Engl J Med. 2007; 356(15):1503-16. [PubMed: 17387127] 


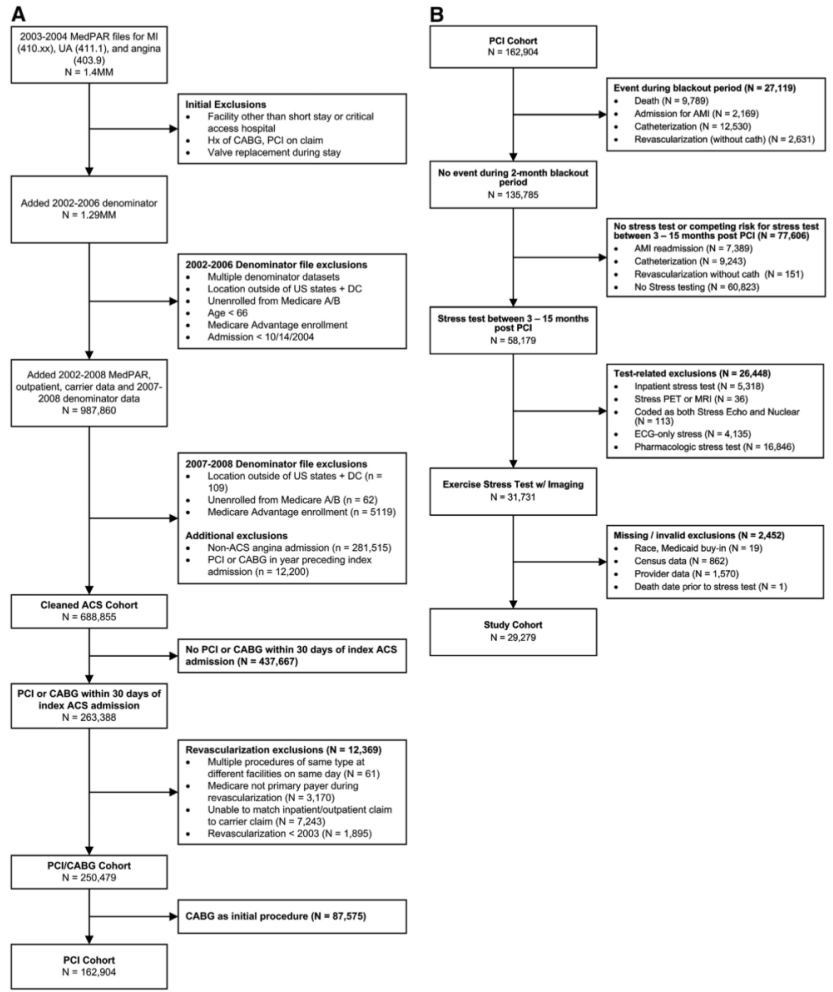

Figure 1.

$\mathbf{A}$ and $\mathbf{B}, \mathrm{A}$ cohort selection diagram illustrating a process by which study cohort was identified. Included patients were those receiving an outpatient exercise nuclear or echocardiography stress test not preceded by another cardiac event. 


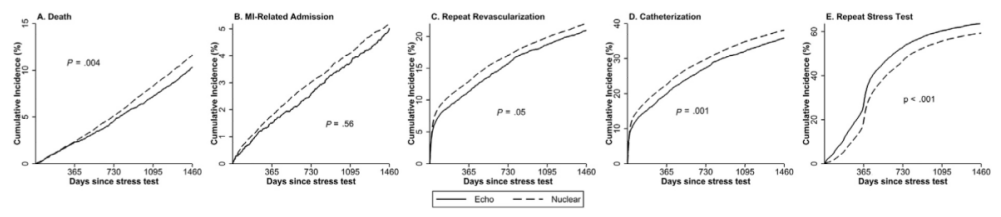

Figure 2.

Unadjusted outcomes by stress test imaging modality. Curves illustrate unadjusted cumulative incidence of outcomes, based on time since stress testing. $P$ values are for comparison of nuclear versus echocardiography using Gray test. 

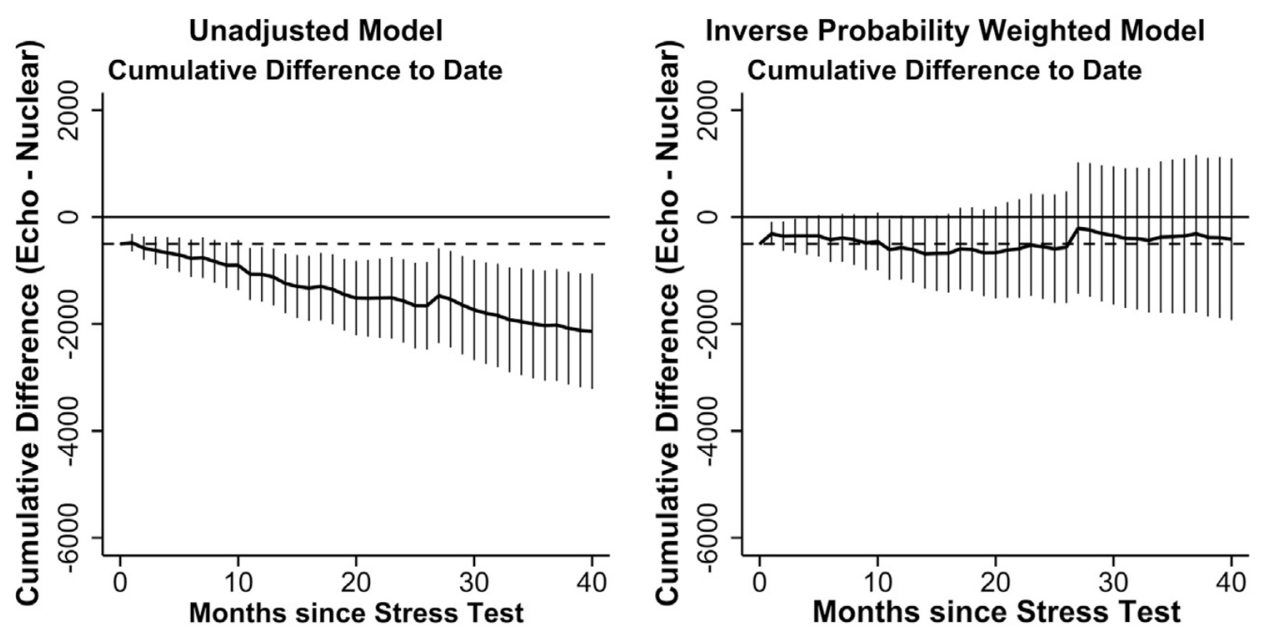

Figure 3.

Unadjusted and adjusted incremental total Medicare payments. A comparison of exercise echocardiography and exercise nuclear. Dotted lines indicate incremental difference in cost accruing during the day of initial stress testing. 

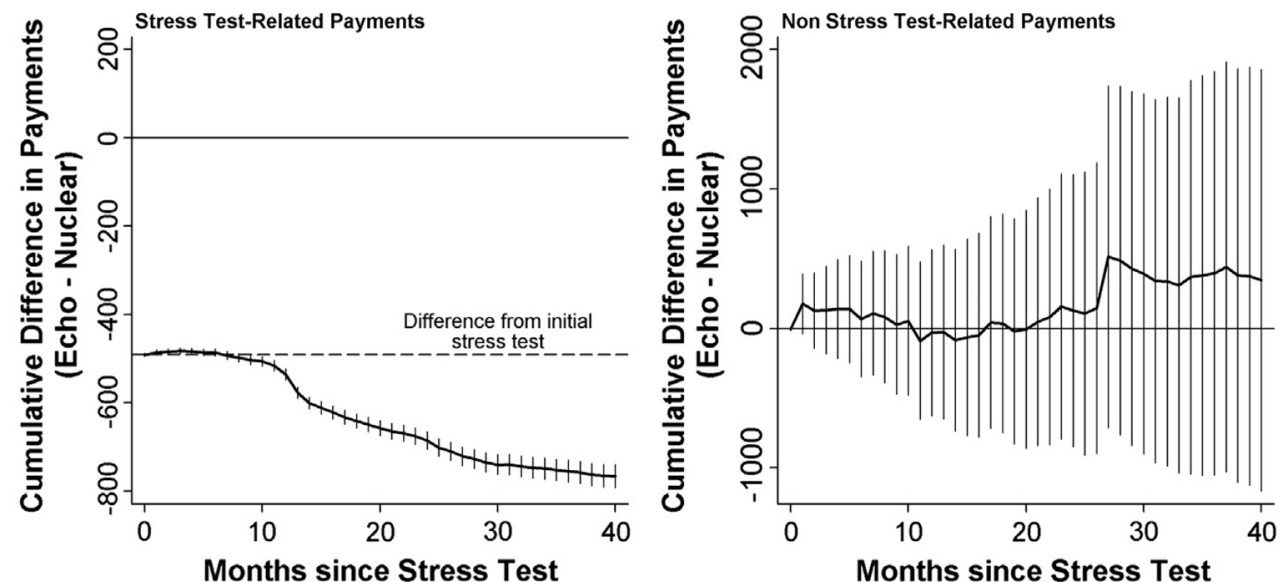

Figure 4.

Inverse propensity weighted-adjusted cumulative incremental costs. A comparison of echocardiography and nuclear imaging with costs stratified by stress test-related versus all other. 


\section{Table I}

Selected baseline patient characteristics

\begin{tabular}{|c|c|c|c|c|}
\hline & Overall $(n=29,279)$ & $\begin{array}{l}\text { Exercise nuclear }(n= \\
\quad \mathbf{2 4 , 7 3 7})\end{array}$ & $\begin{array}{l}\text { Exercise echo }(n= \\
4542)\end{array}$ & $P$ \\
\hline \multicolumn{5}{|l|}{ Demographics } \\
\hline Age (y), median (25th-75th percentile) & $74.0(70.0-78.0)$ & $74.0(70.0-78.0)$ & $74.0(70.0-78.0)$ & .45 \\
\hline Female gender $(\%)$ & 42.5 & 42.6 & 42.3 & .71 \\
\hline Nonwhite race (\%) & 6.2 & 6.1 & 6.8 & .07 \\
\hline State Medicaid buy-in (\%) & 6.9 & 6.8 & 7.3 & .27 \\
\hline \multicolumn{5}{|l|}{ ZIP code characteristics (\%) } \\
\hline $\begin{array}{l}\text { Household income }(\$ 10000 \mathrm{~s}) \text {, median (25th- } \\
\text { 75th percentile) }\end{array}$ & $4.1(3.4-5.3)$ & $4.1(3.4-5.3)$ & $4.2(3.4-5.4)$ & .02 \\
\hline $\begin{array}{l}\text { Percentage living in poverty, median (25th-75th } \\
\text { percentile) }\end{array}$ & $8.8(5.2-13.8)$ & $8.8(5.1-13.8)$ & $9.0(5.5-13.8)$ & .02 \\
\hline $\begin{array}{l}\text { Percentage with college education, median } \\
\text { (25th-75th percentile) }\end{array}$ & $26.9(19.3-39.6)$ & $26.7(19.1-39.2)$ & $28.0(20.1-41.4)$ & $<.001$ \\
\hline Census region $(\%)$ & & & & $<.001$ \\
\hline New England & 5.6 & 5.6 & 6.1 & \\
\hline Middle Atlantic & 14.2 & 14.7 & 11.4 & \\
\hline East North Central & 20.2 & 20.6 & 18.5 & \\
\hline West North Central & 10.1 & 9.6 & 12.6 & \\
\hline South Atlantic & 21.5 & 23.3 & 11.8 & \\
\hline East South Central & 6.4 & 6.9 & 3.7 & \\
\hline West South Central & 8.4 & 8.3 & 8.6 & \\
\hline Mountain & 5.1 & 4.4 & 8.6 & \\
\hline Pacific & 8.5 & 6.7 & 18.8 & \\
\hline \multicolumn{5}{|l|}{ Clinical characteristics (\%) } \\
\hline ACS diagnosis & & & & .23 \\
\hline UA & 45.1 & 45.0 & 45.6 & \\
\hline NSTEMI & 25.7 & 25.9 & 24.7 & \\
\hline STEMI & 29.2 & 29.1 & 29.7 & \\
\hline Multivessel PCI & 19.5 & 19.5 & 19.4 & .82 \\
\hline Stent insertion & 97.1 & 97.1 & 96.8 & .22 \\
\hline Any DES insertion & 45.2 & 44.9 & 47.0 & .01 \\
\hline Any BMS insertion & 53.5 & 53.9 & 51.1 & $<.001$ \\
\hline \multicolumn{5}{|l|}{ Selected comorbidities } \\
\hline Heart failure & 12.6 & 13.0 & 10.5 & $<.001$ \\
\hline Valve disease & 8.1 & 8.1 & 7.7 & .31 \\
\hline Pulmonary circulation disease & 2.0 & 2.0 & 1.6 & .04 \\
\hline Peripheral vascular disease & 12.7 & 12.9 & 11.6 & .02 \\
\hline Neurologic disease & 2.5 & 2.5 & 2.2 & .25 \\
\hline Chronic lung disease & 19.1 & 19.4 & 17.1 & $<.001$ \\
\hline Diabetes: uncomplicated & 21.7 & 22.1 & 19.7 & $<.001$ \\
\hline Diabetes: complicated & 5.3 & 5.5 & 4.2 & $<.001$ \\
\hline
\end{tabular}




\begin{tabular}{|c|c|c|c|c|}
\hline & Overall $(n=29,279)$ & $\begin{array}{l}\text { Exercise nuclear }(n= \\
24,737)\end{array}$ & $\underset{4542)}{\text { Exercise echo }(n=}$ & $P$ \\
\hline Hypothyroidism & 12.4 & 12.4 & 12.4 & .97 \\
\hline Renal failure & 2.8 & 2.8 & 2.6 & .33 \\
\hline Liver disease & 1.7 & 1.8 & 1.3 & .02 \\
\hline Obesity & 5.8 & 5.9 & 5.0 & .02 \\
\hline Hypertension & 77.3 & 77.7 & 75.1 & $<.001$ \\
\hline Recent stroke & 6.6 & 6.7 & 6.0 & .06 \\
\hline \multicolumn{5}{|l|}{ Facility characteristics } \\
\hline Hospital ownership (\%) & & & & .05 \\
\hline Nonprofit & 80.6 & 80.5 & 81.3 & \\
\hline For-profit & 10.1 & 10.3 & 9.1 & \\
\hline Government & 9.3 & 9.2 & 9.6 & \\
\hline Major medical school affiliation (\%) & 32.0 & 31.9 & 32.7 & .27 \\
\hline $\begin{array}{l}\text { No. of CMS-authorized beds, median (25th-75th } \\
\text { percentile) }\end{array}$ & $411(285-597)$ & $420(289-606)$ & $375(269-537)$ & $<.001$ \\
\hline \multicolumn{5}{|l|}{ Prestress test characteristics } \\
\hline $\begin{array}{l}\text { Medicare payments } 1-60 \mathrm{~d} \text { pretest }(\$) \text {, median } \\
\text { (25th-75th percentile) }\end{array}$ & $274(102-687)$ & $276(104-691)$ & $258(92-667)$ & $<.001$ \\
\hline $\begin{array}{l}\text { Calendar time }(d)(1=3 / 1 / 2003) \text {, median }(25 \text { th- } \\
\text { 75th percentile) }\end{array}$ & $408(247-598)$ & $402(246-598)$ & $430(257-600)$ & $<.001$ \\
\hline $\begin{array}{l}\text { Time from PCI to stress test (d), median (25th-75th } \\
\text { percentile) }\end{array}$ & $180(112-273)$ & $181(113-273)$ & $177(107-274)$ & .04 \\
\hline Stress test during blackout period (\%) & 20.2 & 19.9 & 21.8 & .005 \\
\hline
\end{tabular}

NSTEMI, Non-ST-elevation myocardial infarction; STEMI, ST-elevation myocardial infarction; UA, unstable angina; DES, drug-eluting stent; $B M S$, bare-metal stent. 


\section{Table II}

Unadjusted and adjusted cause-specific hazards models (echocardiography vs nuclear imaging)

\begin{tabular}{lll}
\hline & Unadjusted model & IPW model \\
\hline Entire study period & $0.88(0.81-0.96)$ & $0.99(0.90-1.09)$ \\
Death & $0.96(0.84-1.09)$ & $1.01(0.87-1.17)$ \\
Myocardial infarction & $0.93(0.87-0.99)$ & $0.94(0.87-1.01)$ \\
Revascularization & $0.91(0.87-0.96)$ & $0.93(0.88-0.98)$ \\
Diagnostic angiography & $1.12(1.07-1.16)$ & $1.07(1.03-1.12)$ \\
Second stress test & & \\
Days 1-90 after stress testing & & $0.87(0.76-0.98)$ \\
Revascularization & $0.84(0.75-0.94)$ & $0.88(0.80-0.97)$ \\
Diagnostic angiography & $0.85(0.78-0.93)$ & $2.60(2.19-3.10)$ \\
Second stress test & $2.78(2.37-3.26)$ & \\
Days 91+ after stress testing & & $0.99(0.90-1.08)$ \\
Revascularization & $0.98(0.90-1.07)$ & $0.96(0.89-1.03)$ \\
Diagnostic angiography & $0.95(0.89-1.01)$ & $1.03(0.98-1.07)$ \\
\hline Second stress test & $1.06(1.02-1.11)$ & \\
\hline
\end{tabular}

